\title{
FACTORIAL ANALYSIS OF BALANCED FOUR- AND HIGHER-POINT LINKAGE TESTS
}

\author{
W. F. BODMER and P. A. PARSONS \\ Department of Genetics, University of Cambridge
}

Received 12.xii. 58 :

\section{INTRODUCTION}

Bodmer and Parsons (1959) and Bodmer (1959) have given a method for a comprehensive analysis of balanced multi-point linkage experiments using the techniques of factorial experimentation. The cases of two and three points were discussed in detail. In this paper the method is extended to four- and higher-point data. The problem of distinguishing between additive and multiplicative systems, considered by Bodmer (1 959), is discussed in relation to four-point data in the house mouse published by Parsons (1958).

\section{FOUR-POINT DATA}

The data from a complete $n$-point backcross linkage test can, in general, be set out in a $2^{n-1} \times 2^{n-1}$ latin square whose elements are the observations for pairs of complementary genotypes. As for the threepoint test, the columns correspond to the modes of gamete formation,

TABLE I

Scheme for the analysis of variance of a four-point test

Latin square of sums

D.F.

Parental heterozygote (rows)

Recombination (columns)

Two-factor interactions

Four-factor interaction

Error

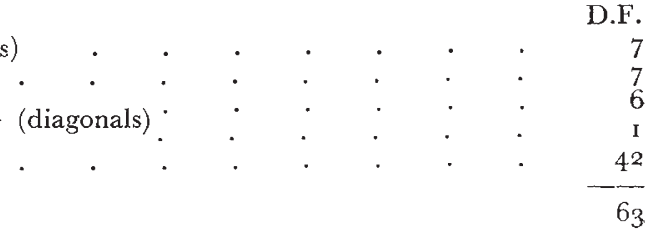

Latin square of differences

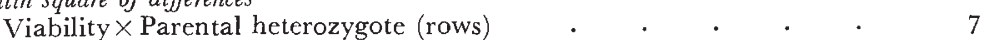

Viability $\times$ Recombination (columns) . . .

Main effects and three-factor interactions with the exception of that

Error represented by the total of the differences square (diagonals) . 7

One of the main effects or three-factor interactions (that which represents the total of the differences square)

Total

the rows to the parental heterozygotes and the diagonals to the $2^{n-1}$ possible pairs of complementary genotypes. Thus the total S.S. (sum of squares) for any complete backcross linkage test consists of 
$\frac{1}{2}$ [S.S. of sums of complementary genotypes] $+\frac{1}{2}[$ S.S. of the differences of complementary genotypes] + a S.S. for the total of the differences. The first two items represent S.S. for latin squares which can be partitioned in the normal way into components for rows, columns, diagonals and an error term, whose meanings are exactly analogous to the meanings of the corresponding components in the three-point test discussed by Bodmer and Parsons (1959).

For a four-point test the data may be set out in an $8 \times 8$ square with 128 classes. The scheme for the analysis of variance is given in table I, and is identical with the three-point situation except for the allocation of viabilities and their interactions.

There are six two-factor interactions and one four-factor interaction. The S.S. for the two- and four-factor interactions is, symbolically,

$$
\begin{aligned}
& \frac{1}{1 \frac{1}{2}}\left[\{(a-\mathrm{I})(b-\mathrm{I})(c+\mathrm{I})(d+\mathrm{I})\}^{2}+\{(a-\mathrm{I})(b+\mathrm{I})(c-\mathrm{I})(d+\mathrm{I})\}^{2}\right. \\
& \quad+\{(a-\mathrm{I})(b+\mathrm{I})(c+\mathrm{I})(d-\mathrm{I})\}^{2}+\{(a+\mathrm{I})(b-\mathrm{I})(c-\mathrm{I})(d+\mathrm{I})\}^{2} \\
& \quad+\{(a+\mathrm{I})(b-\mathrm{I})(c+\mathrm{I})(d-\mathrm{I})\}^{2}+\{(a+\mathrm{I})(b+\mathrm{I})(c-\mathrm{I})(d-\mathrm{I})\}^{2} \\
& \left.\quad+\{(a-\mathrm{I})(b-\mathrm{I})(c-\mathrm{I})(d-\mathrm{I})\}^{2}\right]
\end{aligned}
$$

$$
=\frac{1}{16}\left[\begin{array}{c}
(a b c d+\mathrm{I})^{2} \\
+(a+b c d)^{2} \\
+(b+a c d)^{2} \\
+(c+a b d)^{2} \\
+(c d+a b)^{2} \\
+(a d+b c)^{2} \\
+(b d+a c)^{2} \\
+(a b c+d)^{2}
\end{array}\right]-\frac{1}{128}\left[\begin{array}{c}
(a b c d+\mathrm{I}) \\
+(a+b c d) \\
+(b+a c d) \\
+(c+a b d) \\
+(c d+a b) \\
+(a d+b c) \\
+(b d+a c) \\
+(a b c+d)
\end{array}\right]
$$

where $a b c d$, etc. represent the total contributions of the corresponding genotypes to the eight modes of gamete formation. This is the diagonals S.S. of the sums square as shown in the analysis of variance.

The interpretation of the diagonals S.S. for the differences square is a little more difficult. The $a b c$ interaction may be written symbolically as,

$$
\begin{aligned}
(a-\mathrm{I})(b-\mathrm{I})(c-\mathrm{I})(d+\mathrm{I}) & \\
=(a b c d-\mathrm{I})+(a-b c d)+(b-a c d)+(c-a b d) & +(c d-a b)+(a d-b c) \\
& +(b d-a c)+(a b c-d)
\end{aligned}
$$

If the differences square is made up by taking the differences between complementary pairs as is shown in this expression, the S.S. for the total of the differences square will represent the $a b c$ interaction. The diagonals S.S. of the differences square can then be shown to represent the four main viability effects and three of the four three-factor interactions, the fourth being the $a b c$ interaction.

Thus the composition of the diagonals S.S. of the differences square depends on the interaction that is represented by the total of the differences square. 


\section{FIVE-POINT DATA}

The data from a complete five-point test may be set out in a $16 \times 16$ square split into complementary pairs giving a total of 5 I 2 classes leaving $5 \mathrm{II}$ D.F. after the restriction that the total observed must equal the total expected. The 5 I I D.F. may be split as in table 2.

\section{TABLE 2}

Scheme for the analysis of variance of a five-point test

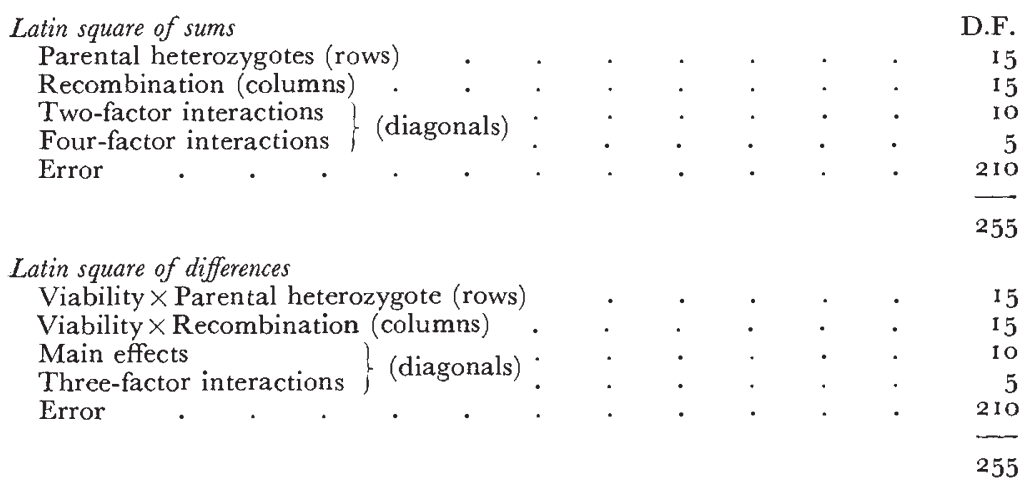

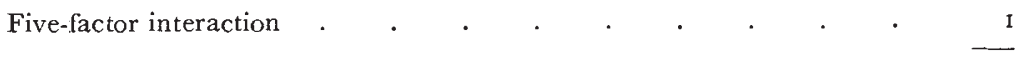
Total . . . . . . . . . 511

In this case, the S.S. of the sum of the differences square is arranged to represent the five-factor interaction $(a-\mathrm{I})(b-\mathrm{I})(c-\mathrm{I})(d-\mathrm{I})(e-\mathrm{I})$, although it could equally well represent a main effect or three-factor interaction.

In the three-point situation discussed by Bodmer and Parsons (1959) the S.S. for the total of the differences square represents the $a b c$ interaction, although the calculation could be equally well done if the sum of the differences square represented one of the three main effects. In the two-point situation, the S.S. of the total of the differences square represents either the main effect of $a$ or of $b$.

This analysis may be extended to data involving six or more factors, although it is unlikely that experiments of this magnitude would be undertaken.

\section{CONFOUNDING IN FOUR- AND HIGHER-POINT DATA}

It was pointed out by Bodmer and Parsons (1959), that, as in agricultural factorial experiments, if we are willing to neglect higherorder interactions, the technique of confounding may be used to reduce the number of heterozygotes needed in a balanced multi-point backcross linkage experiment.

Only interactions involving an even number of factors can be confounded, as the complementary genotypes cannot be separated. 
The general problem of selecting such subsets is complicated, but a scheme for finding them has been devised by Edwards (1958). We shall consider in detail the analysis needed for a confounded four-point test.

Bodmer and Parsons (1959) showed how it was possible to confound the four-factor interaction in a four-point test and so reduce the number of heterozygotes needed from 8 to 4. The data from such a set of four heterozygotes can be set out as in table 3, where a genotype is designated by small letters for the loci at which it is homozygous. The four pairs of complementary genotypes (I, abcd), (bc,ad), $(c d, a b)$ and $(b d, a c)$ are arranged in a latin square whose rows correspond to.

TABLE 3

Genotypes from the four heterozygotes which constitute a balanced set

\begin{tabular}{|c|c|c|c|c|c|c|c|c|}
\hline \multirow{2}{*}{$\begin{array}{c}\text { Parental } \\
\text { heterozygote }\end{array}$} & \multicolumn{8}{|c|}{ Modes of gamete formation } \\
\hline & (o) & (2) & (123) & (13) & $(\mathrm{r})$ & (12) & $(23)$ & (3) \\
\hline$\frac{a b c d}{++++}$ & $\begin{array}{l}a b c d \\
\mathbf{I}\end{array}$ & $\begin{array}{l}c d \\
a b\end{array}$ & $\begin{array}{l}b d \\
a c\end{array}$ & $\begin{array}{l}a d \\
b c\end{array}$ & $\begin{array}{c}a \\
b c d\end{array}$ & $\begin{array}{l}b \\
a c d\end{array}$ & $\stackrel{c}{a b d}$ & $\begin{array}{c}d \\
a b c\end{array}$ \\
\hline$\frac{a b++}{++c d}$ & $\begin{array}{l}c d \\
a b\end{array}$ & $\begin{array}{l}a b c d \\
\mathbf{I}\end{array}$ & $\begin{array}{l}a d \\
b c\end{array}$ & $\begin{array}{l}b d \\
a c\end{array}$ & $\begin{array}{c}b \\
a c d\end{array}$ & $\begin{array}{c}a \\
b c d\end{array}$ & $\begin{array}{c}d \\
a b c\end{array}$ & $\begin{array}{c}c \\
a b d\end{array}$ \\
\hline$\frac{+b+d}{a+c+}$ & $\begin{array}{l}b d \\
a c\end{array}$ & $\begin{array}{l}a d \\
b c\end{array}$ & $\underset{\mathbf{I}}{a b c d}$ & $\begin{array}{l}c d \\
a b\end{array}$ & $\stackrel{c}{a b d}$ & $\begin{array}{c}d \\
a b c\end{array}$ & $\begin{array}{c}a \\
b c d\end{array}$ & $\begin{array}{c}b \\
a c d\end{array}$ \\
\hline$\frac{+b c+}{a++d}$ & $\begin{array}{l}a d \\
b c\end{array}$ & $\begin{array}{l}b d \\
a c\end{array}$ & $\begin{array}{l}c d \\
a b\end{array}$ & $\begin{array}{c}a b c d \\
\mathrm{I}\end{array}$ & $\begin{array}{c}d \\
a b c\end{array}$ & 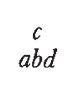 & $\begin{array}{c}b \\
a c d\end{array}$ & $\begin{array}{c}a \\
b c d\end{array}$ \\
\hline
\end{tabular}

the heterozygotes, and columns to the four modes of gamete formation (o), (2), (13) and (123). The remaining pairs of genotypes form a second latin square whose columns are the modes of gamete formation (I), (12), (3) and (23). The total S.S. for data from such an experiment may therefore be split into the S.S. from these two latin squares, which we shall call A and B, say, and the S.S. for the difference between the totals of the squares. This latter is then the confounded degree of freedom. The S.S. for each of A and B may then be split up as in the analysis of the three-point experiment into a latin square of sums, a latin square of differences, and a contribution from the total of the differences square. The interpretation of the components is similar to that considered in previous sections, except that corresponding components from $\mathrm{A}$ and $\mathrm{B}$ have to be combined to make them represent the viability effects and interactions. Thus if the differences squares are arranged so that their total represents the $a b c$-interaction 
we have

$8 \times a b c$ interaction $=(a b c d-\mathbf{I})+(c d-a b)+(a d-b c)+(b d-a c)$

$$
\begin{aligned}
& +(a-b c d)+(b-a c d)+(a b c-d)+(c-a b d) \\
=y_{A}+y_{B}, \text { say } &
\end{aligned}
$$

and $8 \times d$-effect $=(a b c d-\mathrm{I})+(c d-a b)+(a d-b c)+(b d-a c)$

$$
\begin{aligned}
& -[(a-b c d)+(b-a c d)+(a b c-d)+(c-a b d)] \\
& =y_{A}-y_{B}
\end{aligned}
$$

where $y_{A}$ and $y_{B}$ represent the totals of the differences squares from $A$ and $B$. Hence the S.S. from the totals of the differences squares, which is

$$
\frac{1}{32}\left(y_{A}^{2}+y_{B}^{2}\right)=\frac{1}{64}\left(y_{A}+y_{B}\right)^{2}+\frac{1}{64}\left(y_{A}-y_{B}\right)^{2},
$$

is also the S.S. for the $a b c$-interaction and the $d$-effect. In a similar way it can be shown that the S.S. for the diagonals from the two differences squares is the S.S. for the remaining one- and three-factor effects, and the S.S. for the diagonals of the two sums squares is the S.S. for the two-factor interactions. The heterozygote effect will be represented by the S.S. of the totals of the heterozygote contributions, and when subtracted from the S.S. for the rows of the sums squares, leaves a residual representing interaction between viabilities and modes

TABLE 4

Scheme for the analysis of a confounded four-point experiment

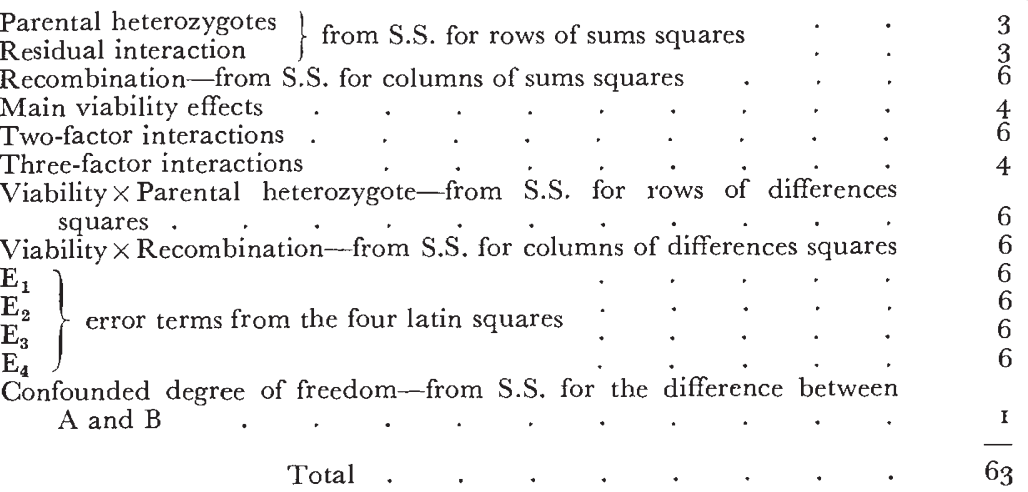

of gamete formation. The S.S. for rows and columns of the differences squares have their usual interpretation as viability $\times$ heterozygote and viability $\times$ recombination interactions and there are four error terms. The resulting scheme for the complete analysis of a confounded four-point experiment is given in table 4 .

\section{NUMERICAL APPLICATIONS TO FOUR-POINT DATA}

Data for a complete four-point backcross linkage test performed on the house mouse were given by Parsons (1958). They involve the factors fuzzy $(f z)$, Splotch $(S p)$, leaden $(l n)$ and polydactyly $(p y)$, of which 
all except the second are recessive. Since we are interested in the effect of $S p$ on viability and this only occurs heterozygously, we must consider the heterozygote $S p+$ as representing the same treatment levels as the recessive homozygotes $f z f z, \ln \ln , p y p y$. The analysis of $\chi^{2}$ on the complete male data is presented in table 5 , where the total of

TABLE 5

Analysis of $\chi^{2}$ of Parsons' $\left({ }_{\left.195^{8}\right)}\right)$ four-point male data for the house mouse

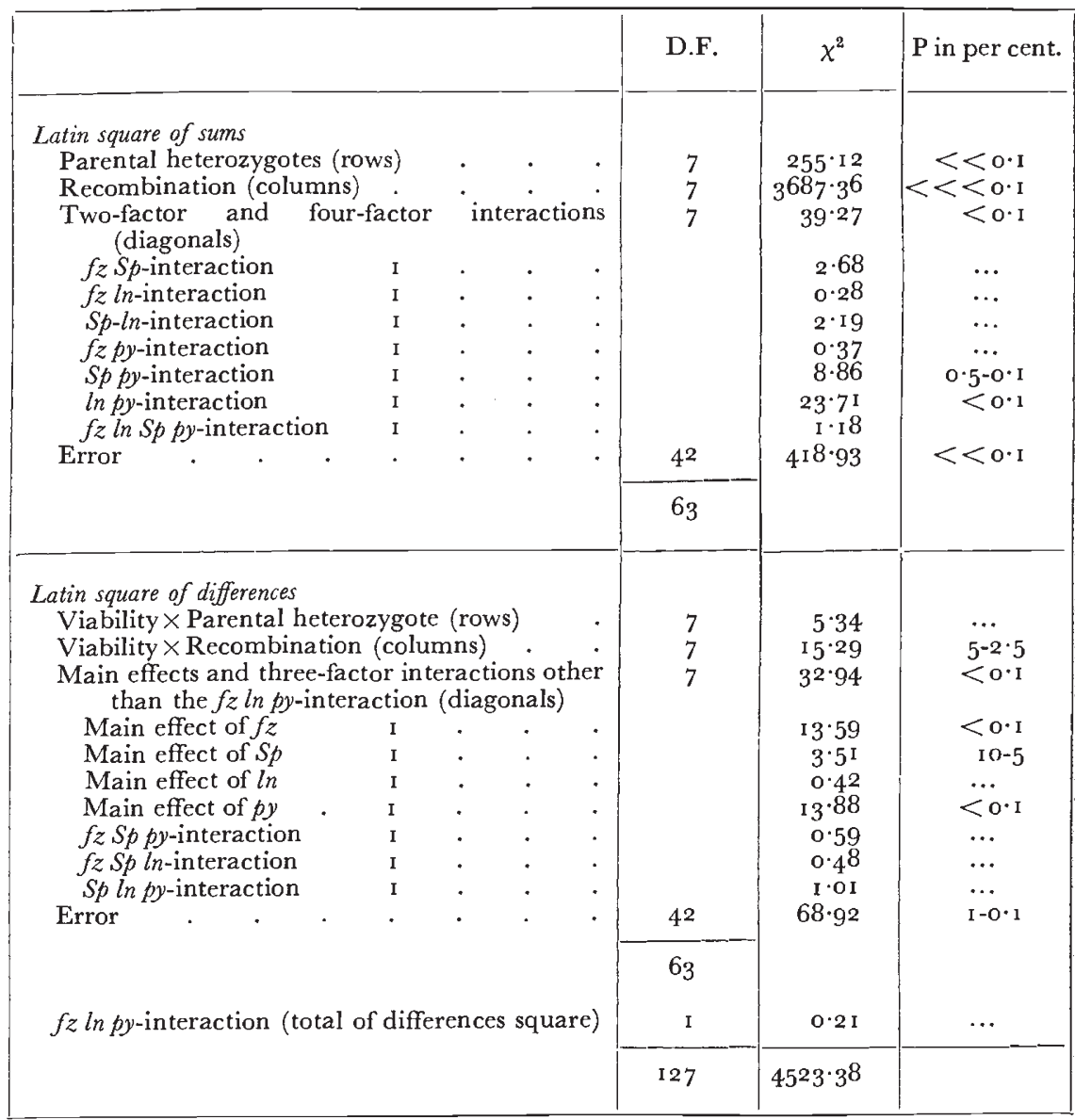

the differences square has been taken to represent the $f z$ ln $p y$ interaction. Only significance levels less than io per cent. are indicated.

By far the largest component is that due to recombination. There is also very severe non-orthogonality indicated by the highly significant parental heterozygote component. Such severe non-orthogonality may seriously affect the terms for viability interactions involving an even number of factors and will in general make the $\chi^{2}$ analysis somewhat inaccurate. This accounts for the significant error term in the sums square, and also for the apparent $S p p y$ and $\ln p y$ interactions. 
Terms in the differences square are less affected by non-orthogonality, although even here the error term is significant as also is the viability $\times$ recombination interaction. This latter represents an effect which could have no simple biological meaning and its significance is almost certainly due to the non-orthogonality. Some confidence can be placed in the significant effects for $f z$ and $p y$. The factor $f z$ is well known to have a somewhat deleterious effect. Polydactyly is not generally associated with viability deficiency, but was, in the stock used by Parsons, not perfectly manifesting. It was shown by Parsons (1958) that the polydactyly effect in these data was almost certainly due to misclassification of the polydactyly and not to any viability disturbance. The problem of estimating recombination in multiple linkage tests with one factor imperfectly manifesting has been considered by Parsons (1957). An examination of the scheme of expectations given for such a situation indicates that the general effect of misclassification on the analysis of $\chi^{2}$ will be indistinguishable from the effect of a corresponding viability deficiency. The $S p$ effect is a little inflated which might be interpreted as an indication of a slight viability deficiency. It is, however, the largest of the remaining six interactions involving an odd number of factors, and so its significance must be modified accordingly. The three-factor interactions are all well below significance, as would be expected.

It has been pointed out that the differences square can be arranged in a number of ways, according to which effect will be represented by its total. It is clear that these will in general result in different values for the rows, columns and error terms of the differences square. Each arrangement will give legitimate measures for viability $\times$ heterozygote and viability $\times$ recombination interactions. However if the error term is significant, usually indicating either severe non-orthogonality, or disturbances not taken into account by the analysis, these components may differ considerably for different arrangements of the differences square, and thus not provide reliable measures of viability $\times$ recombination and viability $\times$ heterozygote interactions.

The data from the four heterozygotes

$$
\frac{f z+\ln p y}{+S p++}, \frac{f z+++}{+S p \ln p y}, \frac{+++p y}{f z S p \ln +} \text { and } \frac{++\ln +}{f z S p+p y}
$$

only, have been analysed as if they represented the outcome of a confounded four-point experiment. The resulting analysis of $\chi^{2}$ is given in table 6 , where as before, the total of the differences square represents the $f z \ln p y$-interaction. It is on the whole similar to that for the complete data. The effect of the non-orthogonality on the two-factor interactions is more severe, but the main effects and three-factor interactions are still not disturbed by the non-orthogonality. The significance of the residual interaction of $3 \mathrm{D}$.F. is also a result of the non-orthogonality. The most striking difference is the non-significance of the py-effect. This is, however, in the same direction as that for the 
complete experiment and a heterogeneity $\chi_{2}^{2}$ comparing the ratio of polydactyls to normals for the two halves of the experiment gives only I $\cdot 42$.

It is clear that in order to obtain more reliable measures of the viability effects and interactions, some sort of logarithic transformation is needed. The logarithmic transformation cannot be applied to the data as they stand, because of the small numbers of observations in some of the classes. However, if we take the logarithms of the

TABLE 6

Analysis of a confounded four-point experiment based on Parsons' (1958) data

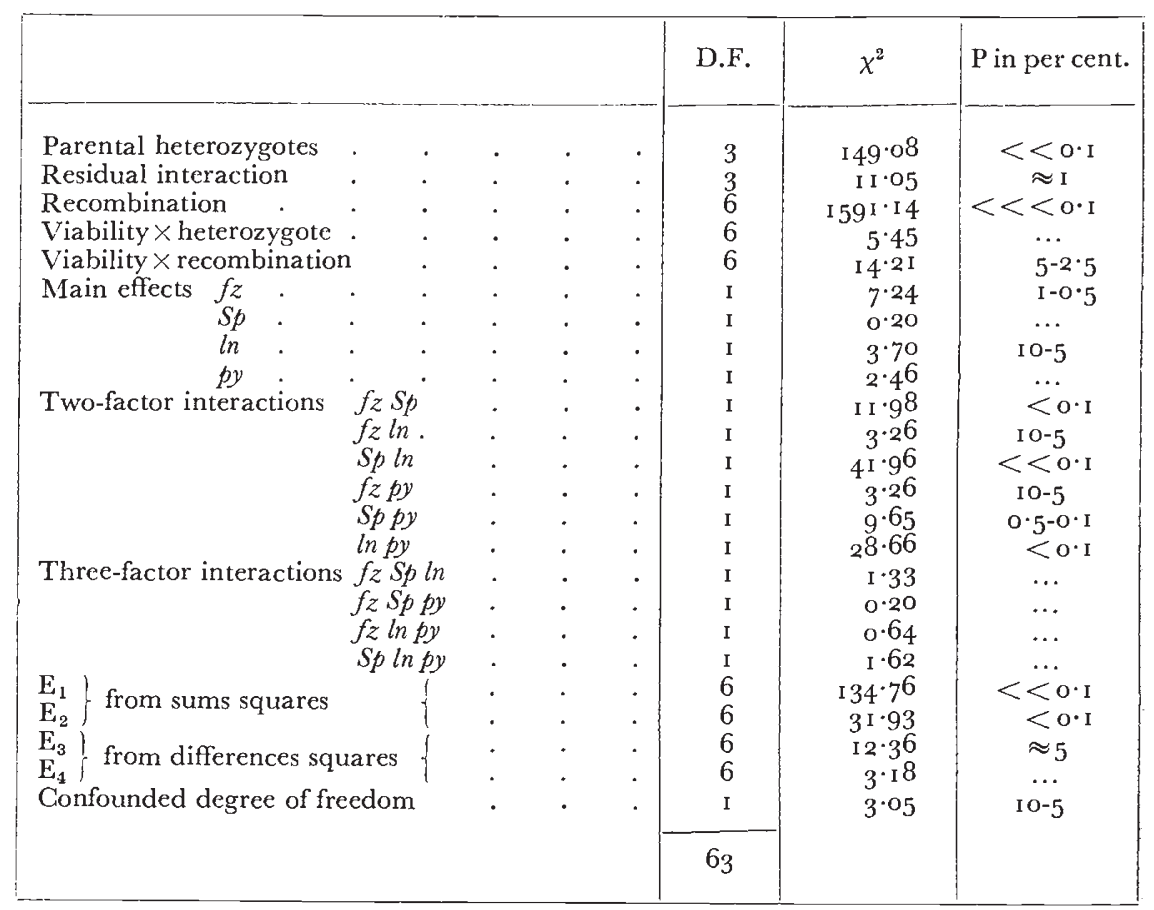

totals observed for each genotype, we will at least obtain unbiased measures of multiplicative effects involving an odd number of factors. Thus in terms of logarithms the $f z$-effect, for example, is

$\frac{1}{8}[\log f z+\log f z S p+\log f z \ln +\log f z p y+\log f z S p \ln +\log f z S p p y$ $+\log f z \ln p y+\log f z S p \ln p y-\log S p-\log \ln -\log p y-\log S p \ln$ $-\log S p p y-\log \ln p y-\log S p \ln p y-\log$ I]

$=\log _{8} \sqrt{\frac{f z \cdot f z S p \cdot f z \ln \cdot f z p y \cdot f z S p \ln \cdot f z S p p y \cdot f z \ln p y \cdot f z S p \ln p y}{S p \cdot \ln \cdot p y \cdot S p \ln \cdot S p p y \cdot \ln p y \cdot S p \ln p y \cdot \mathrm{I}}}$

where $f z, S p$, etc. and I represent the totals observed for the corresponding genotypes. The antilog. of this will be the multiplicative effect of $f z$. Using Fisher's (1925-54) approximate formula for the variance of a statistic expressed as a function of the observed frequencies, 
it is easily seen that the variance of each of the logarithmic multiplicative effects is $\frac{1}{64} \times$ the sum of the reciprocals of the genotype totals. If logarithms are taken to the base ten, this must be multiplied by $\left(\log _{\mathrm{e}} \text { IO }\right)^{2}$. The square of each effect divided by this variance provides an approximate $\chi_{1}^{2}$ for measuring the significance of the effect. These $\chi_{1}^{2}$ 's are shown in table 7 together with the multiplicative effects expressed as a percentage of standard. The results are very similar to those obtained by the analysis on the original data, and still show a

TABLE 7

Tests for multiplicative viability effects in Parsons' (r958) four-point data

\begin{tabular}{|c|c|c|c|c|c|c|c|c|}
\hline & & & & & & $\begin{array}{l}\text { Multiplicative } \\
\text { effects in } \\
\text { per cent. }\end{array}$ & $\chi^{2}{ }_{1}$ & $P$ in per cent. \\
\hline \multicolumn{9}{|l|}{ Main cffects } \\
\hline$f z$ & . & . & . & . & . & $85 \cdot 63$ & I $5 \cdot 0 \mathrm{I}$ & $<0 \cdot I$ \\
\hline$S p$ & . & . & . & . & . & $9^{2} \cdot 64$ & $3 \cdot 53$ & I O- 5 \\
\hline $\ln \quad$ & . & . & . & . & . & IO3. I & 0.59 & $\ldots$ \\
\hline py . & $\cdot$ & · & $\cdot$ & . & $\cdot$ & $86 \cdot 86$ & I $2 \cdot 4$ I & $<0 \cdot I$ \\
\hline \multicolumn{9}{|c|}{ Two-factor interactions } \\
\hline$f_{z} S p$ & . & . & . & . & . & $9^{2} \cdot 68$ & $3 \cdot 60$ & $10-5$ \\
\hline$f z \ln$ & . & . & . & . & . & $97 \cdot 31$ & 0.44 & $\ldots$ \\
\hline$S p \ln$. & . & . & . & . & . & $93 \cdot 1 \mathrm{I}$ & $3 \cdot 18$ & $10-5$ \\
\hline$f z p y$. & - & . & . & . & . & $102 \cdot 4$ & 0.35 & $\ldots$ \\
\hline$S p p y$ & • & . & . & . & . & I $13 \cdot 2$ & $9 \cdot 6 \mathrm{I}$ & $0 \cdot 5-0 \cdot 1$ \\
\hline $\ln p y$. & • & . & · & • & $\cdot$ & $123 \cdot 0$ & $26 \cdot 72$ & $<0 . \mathrm{I}$ \\
\hline \multicolumn{9}{|c|}{ Three-factor interactions } \\
\hline$f z S p \ln$ & - & . & . & . & $\cdot$ & $96 \cdot 5^{6}$ & $0 \cdot 7^{6}$ & $\cdots$ \\
\hline$f z S p p y$ & . & . & . & . & $\cdot$ & $\mathrm{IO} 4 \cdot \mathrm{I}$ & $0 \cdot 98$ & $\cdots$ \\
\hline$f z \ln p y$ & - & · & $\cdot$ & $\cdot$ & $\cdot$ & 103.9 & 0.90 & $\cdots$ \\
\hline$S p \ln p y$ & · & · & · & $\cdot$ & $\cdot$ & $104 \cdot 8$ & $1 \cdot 37$ & $\cdots$ \\
\hline \multicolumn{9}{|c|}{ Four-factor interaction } \\
\hline$f z S p \ln p y$ & . & . & . & - & - & I05.6 & I $\cdot 85$ & $\cdots$ \\
\hline
\end{tabular}

marked disturbance of the two-factor interactions by the non-orthogonality. The additive effect of $f z$, for example, was -2.92 I 9 relative to an overall mean of 20. IоI6, and the corresponding multiplicative effect is $-2 \cdot 8886$. The similarity between the two analyses shows that the effects are too small for a difference between additive and multiplicative systems to be detectable. The estimate for the fuzzy viability of 85.63 per cent. has a standard error of 3.43 . An examination of the expectations given by Parsons (1957) for situations in which there is misclassification of one factor shows that if the $p y$-effect is entirely due to misclassification it will approximately be an estimate of $(\mathrm{I}-\lambda) /(\mathrm{I}+\lambda)$ where $\lambda$ is the percentage misclassification of $p y$. This gives an estimate for $\lambda$ of $7 \cdot 032 \pm \mathrm{I} \cdot 992$ per cent. which is in close agreement with the value obtained by Parsons (I958) of 6.910 \pm I.959 per cent., by a different method. 
As there is no real evidence for any interactions involving $S p$, it is legitimate to neglect $S p$ and consider the results as if they had been obtained from a three-point experiment involving $f z, l n$ and $p y$. The data are then no longer too sparse for a direct application of the logarithmic transformation and so make possible an investigation of the effects affected by severe non-orthogonality in the analysis on the original data. The analysis of the data considered in this form and after taking logarithms is given in table 8 . It should be noticed that

TABLE 8

Analysis, after taking logarithms, of Parsons' (1958) data considered as a threepoint test involving $\mathrm{f} z, \ln$ and py

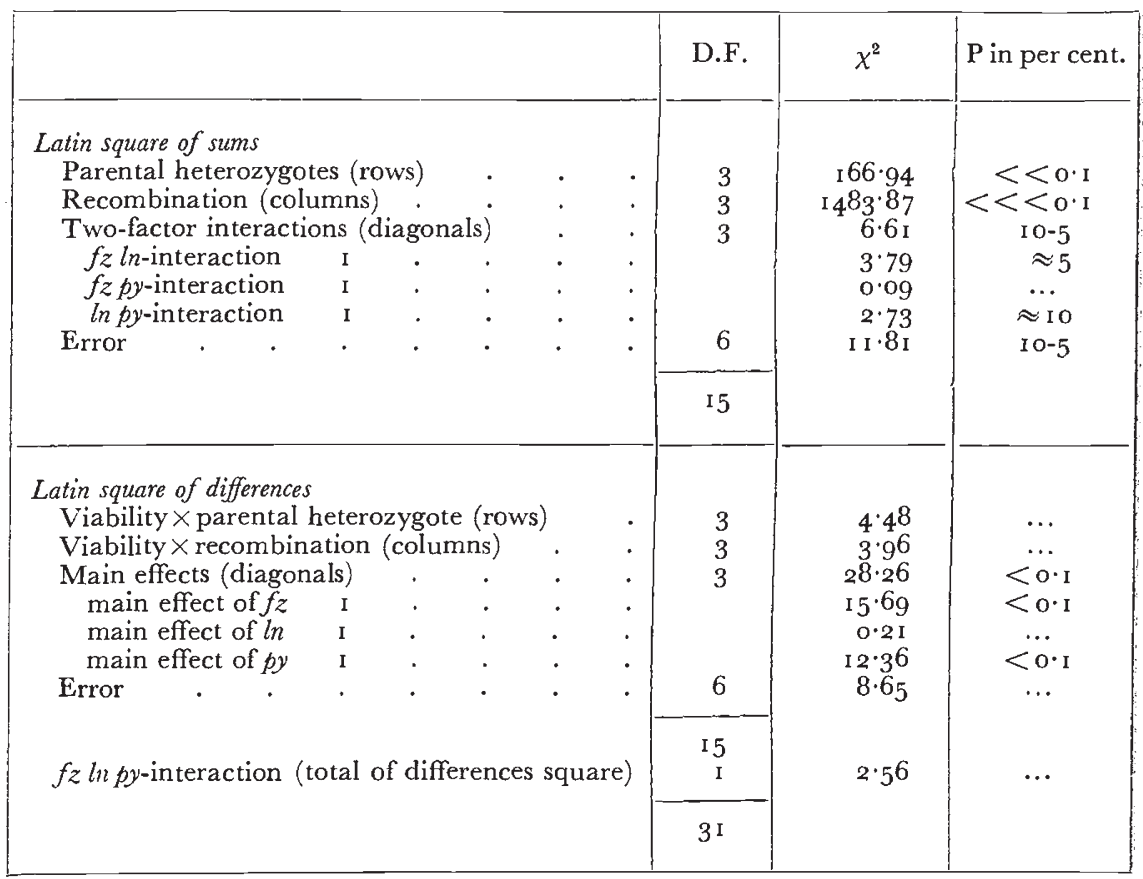

the effects tested are multiplicative effects as defined above. The error term of the sums square and two of the two-factor interactions are still slightly inflated by the non-orthogonality, but the differences. square is no longer affected. There is clearly no viability $\times$ heterozygote or viability $\times$ recombination interaction. The power of the logarithmic transformation in representing the situation on a multiplicative basis and eliminating the effects of the non-orthogonality is quite striking.

\section{DISCUSSION}

The main purpose of this paper has been to apply the factorial analysis of balanced multi-point linkage tests developed for twoand three-point data to four- and higher-point data. The application to the situation where all the possible multiple heterozygotes are used 
as parents in four- and higher-point data is a mere extension of the previous work. The only difficulty is the allocation of the viability effects and interactions.

However, the technique of confounding may be used to reduce the number of heterozygotes needed in four- and higher-point experiments. In a four-point experiment, therefore, by confounding the abcd or four-factor interaction it is possible to reduce the number of heterozygotes needed from eight to four. The analysis of variance for such a balanced set of four is discussed and provides information on all the components that the complete analysis gives with the exception of the confounded abcd interaction. The analysis of balanced sets formed by confounding various four and higher even-order interactions. will be of greater importance in five- and higher-point data where it would be increasingly laborious to make up all the possible multiple heterozygotes.

The techniques developed in this paper are illustrated with an analysis of $\chi^{2}$ of some four-point data in the house mouse given by Parsons (1958). The analysis gives a large $\chi^{2}$ for the parental heterozygote component indicating non-orthogonality and this accounts for large two-factor interactions, viability $\times$ recombination interactions and error terms. In a situation where the error term of the " differences" square is large, the magnitude of the components of the "differences" square may vary considerably according to the viability interaction represented by the total of the differences square. Thus measures of viability $\times$ recombination and viability $\times$ heterozygote interactions will be unreliable under such circumstances.

However, after taking logarithms and analysing the resulting data on the basis of a multiplicative system, the significant interactions and error terms noted above were reduced on the whole to insignificance. Thus the power of the logarithmic transformation in representing the situation on a multiplicative basis is clearly demonstrated. Further evidence of the power of the logarithmic transformation is provided by data of Parsons (1959) in Drosophila melanogaster where many interactions are reduced to insignificance after taking logarithms.

\section{SUMMARY}

I. The factorial analysis of complete four- and five-point linkage data is discussed and the extension to higher-point data indicated.

2. The analysis of a balanced set in a four-point linkage test formed by confounding the four-factor interaction is presented.

3. Four-point data in the house mouse (Parsons, I958) is analysed to illustrate the methods for both the complete data and the balanced set.

4. The data are analysed after taking logarithms as proposed by Bodmer (1959) and it is shown that the logarithmic transformation provides a more realistic picture of the situation. This supports the multiplicative basis of expectations assumed for such experiments. 
Acknowledgment.-One of us (W. F. B.) wishes to acknowledge the receipt of an Agricultural Research Council Studentship during the course of this work.

\section{REFERENCES}

BODMER, W. F. 1959. Multiplicative effects and the logarithmic transformation in the analysis of balanced multi-point linkage tests. Heredity, 13, I57-164.

BODMER, W. F., AND PARSONs, P. A. I959. The analogy between factorial experimentation and balanced multi-point linkage tests. Heredity, ${ }_{3}, I_{45}-I_{5} 6$.

EDWARDS, A. W. F. 1958. Number of mating types required for balance in multipoint linkage programmes. Nature, I8I, 503-504.

FISHER, R. A. 1925-54. Statistical Methods for Research Workers. Oliver and Boyd, Edinburgh.

PARSONS, P. A. 1957. Partial manifestation of a gene in complete three- and higherpoint data. Heredity, II, 21 7-222.

PARSONS, P. A. 1958. A balanced four-point linkage experiment for linkage group XIII of the house mouse. Heredity, 12, 77-95.

PARSONS, P. A. 1959. The dependence of genotypic viabilities on the proportion of co-existing genotypes in Drosophila melanogaster. Heredity, I3, 393-402. 